TITLE:

\title{
Dynamics of electron-plasma vortex in background vorticity distribution
}

\section{$\operatorname{AUTHOR}(S)$ :}

Kiwamoto, Y; Ito, K; Sanpei, A; Mohri, A

\section{CITATION:}

Kiwamoto, Y ...[et al]. Dynamics of electron-plasma vortex in

background vorticity distribution. Physical Review Letters 2000, 85(15):

3173-3176

ISSUE DATE:

2000-10-09

URL:

http://hdl.handle.net/2433/50229

RIGHT:

Copyright 2000 American Physical Society 


\title{
Dynamics of Electron-Plasma Vortex in Background Vorticity Distribution
}

\author{
Y. Kiwamoto, ${ }^{1, *}$ K. Ito, ${ }^{1}$ A. Sanpei, ${ }^{1}$ and A. Mohri ${ }^{2}$ \\ ${ }^{1}$ Department of Fundamental Sciences, Faculty of Integrated Human Studies, Kyoto University, Yoshida Nihonmatsu-cho, \\ Sakyo-ku, Kyoto 606-8501, Japan \\ ${ }^{2}$ The Institute of Physical and Chemical Research, 2-1 Hirosawa, Wako 351-0106, Japan
}

(Received 24 January 2000; revised manuscript received 5 June 2000)

\begin{abstract}
Dynamics of a point vortex in interaction with a broad profile of background vorticity is studied experimentally by using an electron plasma. The observed motion of the vortex compares favorably with a recently proposed theoretical model [D. A. Schecter and D. H.E. Dubin, Phys. Rev. Lett. 83, 2191 (1999)]. Perturbations in the background distribution in the wake of the spiral orbit of the vortex amount to several tens of percent and are considered to be a major reason for deviations of the observation from the linear theoretical model.
\end{abstract}

PACS numbers: 52.25.Wz, 47.32.Cc

The macroscopic dynamics in an electron plasma, trapped axially by an electrostatic potential and radially by a strong homogeneous magnetic field, is isomorphic to that of two-dimensional (2D) inviscid fluid, as long as the axial bouncing motion of the electrons is much faster than the transverse $\mathbf{E} \times \mathbf{B}$ drift motion of their guiding centers [1-3]. In this analogy, the $2 \mathrm{D}$ electron fluid is incompressible and the vorticity $\zeta$ is related to the plasma density $n$ and the magnetic field strength $B_{0}$ by $\zeta=e n / \epsilon_{0} B_{0}$, while the streamlines conform to the equipotential surfaces.

Many features of vortex dynamics in 2D Euler fluids have been examined experimentally by using electron plasmas confined in Malmberg traps [4]. Mutual interaction of vortex patches is among extensively studied topics [5-7]. It has been pointed out recently that a background vorticity plays an essential role in the relaxation of 2D turbulence toward an ordered state composed of eminent discrete vortices [8] and in the accelerated merging of remotely separated vortices [9]. In this Letter, we study 2D dynamics of a single point vortex interacting with a large vortex of extended distribution or a background vorticity as an elementary process of the above observations. This configuration is relevant also as a model of an atmospheric vortex moving in a rotating planet [10-12]. A special emphasis is made on comparison with a recently proposed theoretical model [13] because it solely accounts for most of the observations in this experimental study.

In the experiment the point vortex is a string of $N_{v}$ electrons with the half-maximum diameter of $0.5 \mathrm{~mm}$ and length $L=235 \pm 5 \mathrm{~mm}$ confined radially by a homogeneous magnetic field of strength $B_{0}=0.048 \mathrm{~T}$ at maximum. To reduce the spatial variation of the axial length of the strings, we form a Malmberg-type potential distribution by grounding the axially aligned 11 conducting cylinders with $64 \mathrm{~mm}$ inner diameter and by negatively biasing the end-plug cylinders as shown in Fig. 1 [4]. The total circulation of the point vortex is evaluated as $\Gamma_{v}=e N_{v} / \epsilon_{0} B_{0} L$, where $\epsilon_{0}$ is the dielectric constant in vacuum. We generate the vortex strings by injecting elec- trons for $\approx 5 \mu \mathrm{s}$ from a selected element in the array of 19 impregnated-tungsten cathodes and trap them by gated biasing of the end-plug $[9,14]$. The current and energy of electrons emitted from each cathode can be varied independently as well as the timing of the emission.

We establish background vorticity distributions, as shown in Fig. 2(a), by repeating a few hundred times the cycle of injection-trapping-relaxation of electron strings and by letting the mixed state of the plasma relax further for additional $1 \mathrm{~s}$. Through appropriate adjustment of the cathode selection and the stacking sequence we produce different shapes of convex distribution of the background vorticity that by itself remains unchanged for additional $0.1 \mathrm{~s}$. From this profile a concave distribution is created by partially dumping the trapped electrons axially through the injection-side end cylinder. A point vortex is injected immediately after this process.

The measurements are made by dumping the whole electrons through the end cylinder on the other side of the cathode onto a phosphor screen that is biased up to $4.5 \mathrm{kV}$ from the machine ground. The conducting surface of the screen with diameter $50 \mathrm{~mm}$ also serves as an electron collector to monitor the total number of electrons $N$.

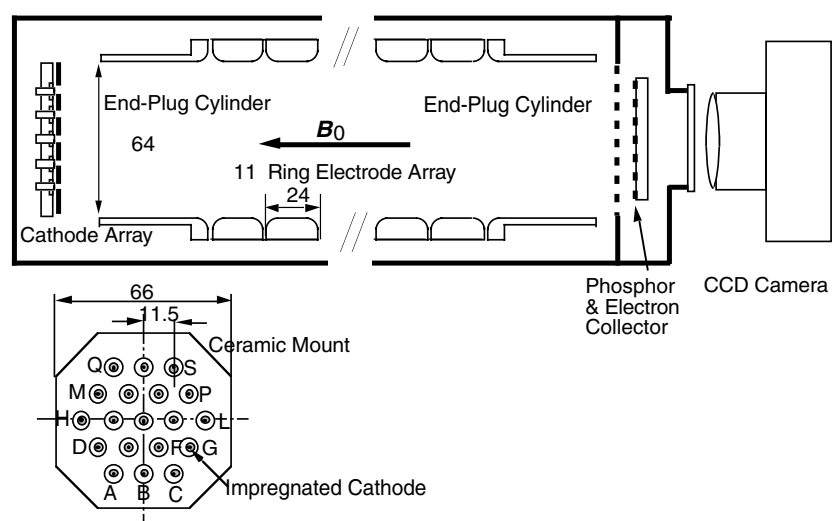

FIG. 1. Schematic configuration of electron emitter array and the plasma trap. The cathodes are labeled $A$ to $S$, to which the point vortices are referred to as sources. 


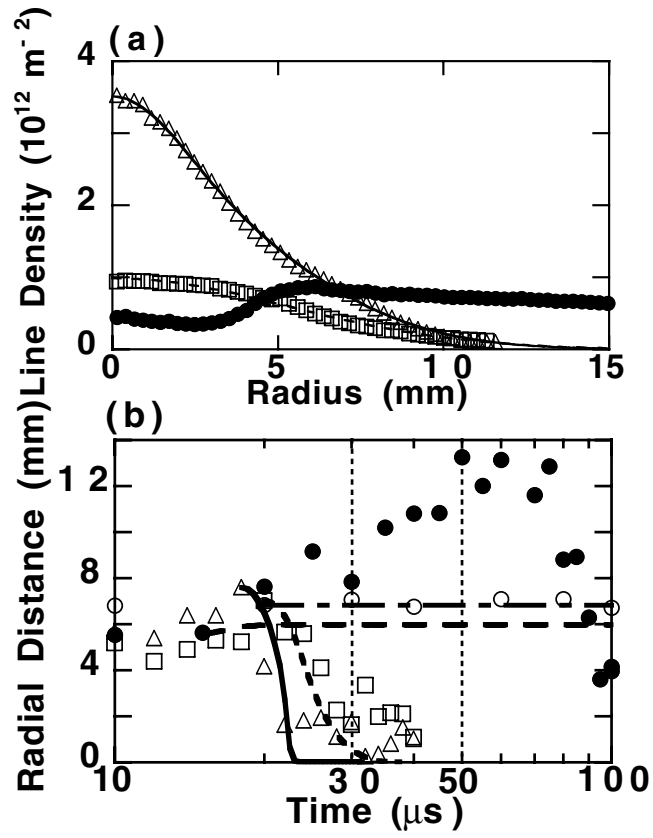

FIG. 2. (a) The radial profile of the line-integrated density distribution of the background electrons. $N_{b} / 10^{8}=3.5(\triangle)$, $1.8(\square)$, and $8.0(\bigcirc)$. (b) The radial distance of a point vortex $F\left(N_{F}=1.2 \times 10^{7}\right)$ from the background center is plotted as a function of time, for radially decreasing profiles $(\triangle, \square)$, for a hollow profile $(\mathbf{)})$, and for the vacuum case $(\bigcirc)$. The curves are calculated from Eq. (1) starting from the position giving the best fit to the initial-phase orbits.

The luminosity distribution on the screen is detected with a charge-coupled-device $(\mathrm{CCD})$ camera with $512 \times 512$ pixels and recorded in a computer for numerical analyses. We have confirmed a good linear relationship between $N$ and the luminosity integrated over the screen.

Using the relationship we obtain the transverse distribution of the line density of the plasma integrated along the magnetic field, and determine an analytical distribution function best fitting to the observed luminosity distribution. For convenience of numerical analyses we employ a superposition of $\exp [f(r)]$ as a fitting function, where $f(r)$ is a polynomial of the radius $r$. The dots in Fig. 2(a) exhibit the azimuthally averaged line density of the background plasma as determined from the luminosity distribution, while the curves represent the best-fitting functions. The electron temperature is about $1 \mathrm{eV}$.

The orbit of a point vortex injected at $t=10 \mu \mathrm{s}$ from the cathode $F$ is plotted in Fig. 2(b) as a function of time for different profiles of the background vorticity. In vacuum $(\bigcirc)$ the point vortex continues orbiting around the machine axis [9]. When the background distribution decreases radially ( $\triangle$ and $\square$ ), the vortex moves toward the center of the background vorticity. When the vortex is injected inside the ridge of a concave distribution ( $)$, it follows the ridge that moves and asymmetrically deforms due to diocotron instability associated with the background distribution [1-3] and tends to settle down on the ridge. A noticeable overshoot of the vortex orbit is attributed to the motion of the background vortex. When the point vortex (from $L$ ) is placed outside the ridge, it moves inward and shows a similar orbit. In either case the point vortex does not cross the ridge. These observations are qualitatively consistent with a theoretical model proposed recently by Schecter and Dubin [13], stating that a point vortex moves up the hill of background vortex. The orbits expected from the model are plotted in solid (for $\triangle$ ), dotted (for $\square$ ), dashed (for $\bullet$ ), and dash-dotted (for $\bigcirc$ ) lines.

According to a slab model by Schecter and Dubin [13], the radial velocity of a point vortex in a background vorticity distribution $\zeta_{b}$ is given by

$$
\frac{d r}{d t}=\frac{\Gamma_{v}}{2 \pi} \frac{d \zeta_{b} / d r}{|A|} \ln \left(\frac{c r}{l}\right) \arctan \left(\frac{|A| t}{2}\right),
$$

where $A=-r d \Omega_{b}(r) / d r$ is the shear relative to the local rigid rotation $\Omega_{b}(r)$ of the background vortex, $l=$ $\left(\Gamma_{v} / 2 \pi|A|\right)^{1 / 2}$ is the radius of the stagnation zone around the point vortex with a circulation of $\Gamma_{v}$, and $c$ is a number of order 1 . In the following we put the logarithmic factor as 1 to avoid an unphysical lower limit at $r=l / c$ [13].

Figure 3 shows the observed motion of a point vortex with different $\Gamma_{v}$ starting from the arrow-marked radius in the same initial background profile as shown in the inset. The vortex is injected at $t=10 \mu \mathrm{s}$, and starts to move freely on disconnection from the cathode at $t \approx 15 \mu \mathrm{s}$ as indicated by an arrow. The latter timing is determined as a stepwise reduction of the charge dumped on the collecter screen. The observation shows that the radial velocity of the vortex $F$ increases with $\Gamma_{v} \propto N_{F}=(1.6-17) \times 10^{6}$ as expected from Eq. (1). By introducing experimental parameters into Eq. (1), we calculate the vortex orbits as shown in Fig. 3. It indicates that on the whole the theory can describe the observed orbits even quantitatively. Though Eq. (1) does not lead to oscillatory orbits, they

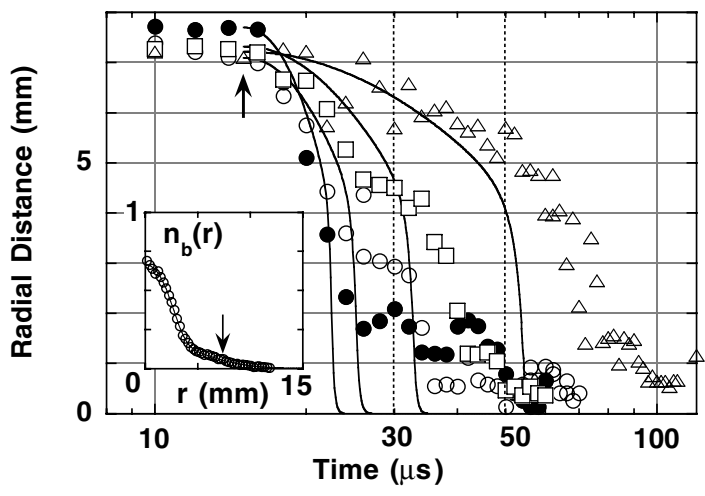

FIG. 3. Radial distance of a point vortex $F$ from the background center is plotted against time after injection at $t=$ $10 \mu \mathrm{s} . \quad N_{F} / 10^{6}=1.6(\triangle), 4.4(\square), 8.7(\bigcirc), 17(\bigcirc)$. The inset shows the common profile of the initial background in unit of $10^{13} \mathrm{~m}^{-3}$ with $N_{b}=7.8 \times 10^{7}$. The curves attached to the dots denote the orbits calculated from Eq. (1). 


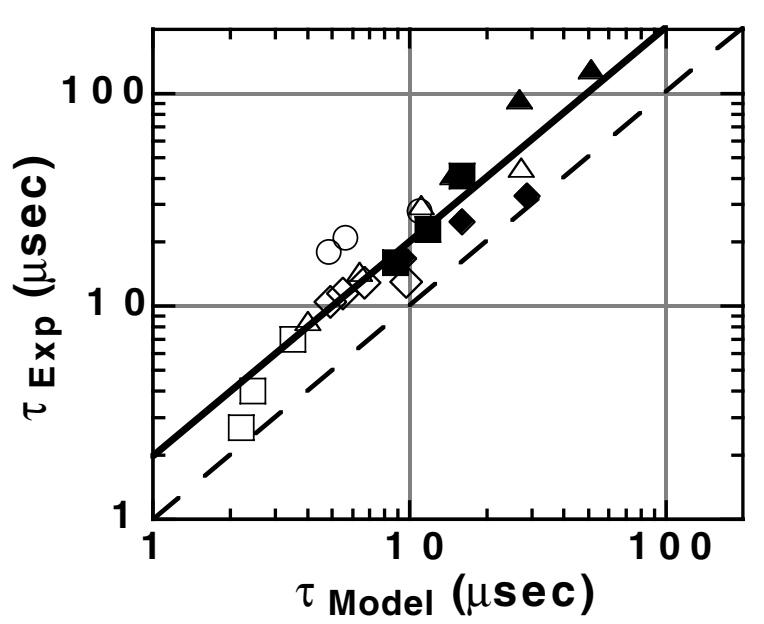

FIG. 4. The observed time $\tau_{\exp }$ is plotted against the theoretical time $\tau_{\text {model }}$. Here $N_{b} / 10^{8}=0.78(\triangle), 1.8(\diamond), 3.5(\square)$, $7.0(\bigcirc)$. The open (closed) symbols denote the data with the vortex $F(L)$ with different values of $N_{F}\left(N_{L}\right) / 10^{6}=1.6-17$. For oscillatory orbits the $10 \%$ points are determined by extrapolating the initial track.

are a natural consequence of the background reaction in a Euler fluid as discussed later.

In Fig. 4 we directly compare the observations with predictions of the model by plotting the times, $\tau_{\exp }$ and $\tau_{\text {model }}$, required for the vortex to move from the position at $90 \%$ of the initial radial distance to the $10 \%$ point toward the center of the background. The latter point is determined by smoothly extrapolating the initial trajectory before the first bounce to remain in the framework of the theory. The plots are collected from data obtained with different experimental parameters including circulation strength $\left(\propto N_{v}, N_{b}\right)$, and initial location of point vortices $(F$ and $L)$ and different profiles of the background vorticity $\left[\propto n_{b}(r)\right]$ with $d \zeta_{b}(r) / d r<0$. The almost linear relation $\tau_{\text {exp }} \approx 2 \tau_{\text {model }}$, as denoted by the solid line, indicates that the model equation (1) agrees quantitatively with experiments. The deviations within a factor of 2 may be reduced by properly treating the logarithmic factor in Eq. (1) or by considering nonlinear effects associated with large $\delta \zeta_{b}$ as mentioned below.

Figure 5 shows frames of the CCD images of the vorticity distribution at different times. Here a point vortex $F$ with $N_{F}=1.7 \times 10^{7}$ is injected at $t=0$ moves into the background vortex with $N_{b}=3.5 \times 10^{8}$. The upper panels display the total vorticity distribution at $t=0,10$, 12,18 , and $26 \mu \mathrm{s}$, respectively. The darkness is proportional to the vorticity except for saturated images of the point vortex. One can observe that a spiral streak forms behind the point vortex. Such a wake structure has been observed also in the vortex-in-cell simulation [13].

The lower panels show the perturbed part $\delta \zeta_{b}$ of the background vorticity, obtained by subtracting the initial background image from the total image. It is observed that, along the streak, the background vorticity decreases on the inner side and increases on the outer side, namely $\delta \zeta_{b}(r-\delta r)<0<\delta \zeta_{b}(r+\delta r)$. In other words, the vorticity in the background is transferred downhill as the point vortex climbs up inward. Schecter and Dubin discussed this process in terms of the conservation of the total canonical angular momentum. In terms of plasma physics, however, we can draw a more concrete picture of the vortex dynamics.

As in Ref. [13] we take the frame rotating at the local angular velocity of the background plasma, with a local coordinate $(x, y)$ system centered on the vortex as shown in Fig. 6(a). If $d n_{b}(r) / d y<0$, the stream lines coming from the right below carry more electrons to the turning point on the positive $x$ axis than those from the left above. The imbalance of the charge density produces an electric field pointing toward positive $x$, and drives the point vortex by $E \times B$ drift toward negative $y$. The associated transport of the background electrons along the streamlines causes excess density in the region right above of the point vortex and depletion left below. This mechanism

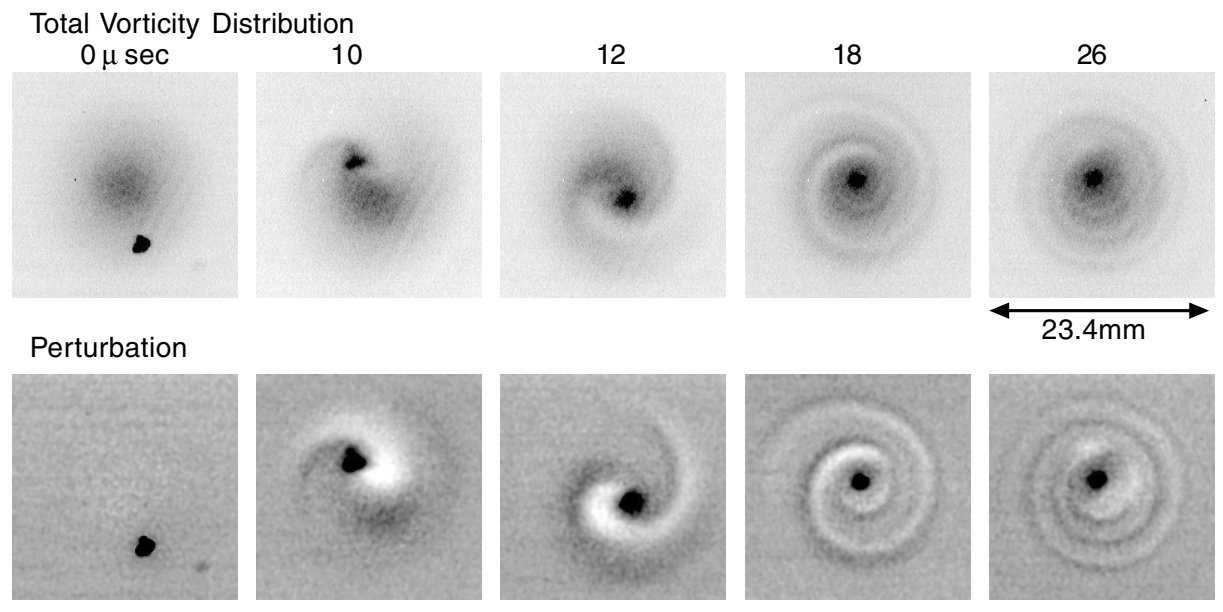

FIG. 5. 2D distribution of the background vorticity at different times. The upper panels exhibit the total vorticity, while the lower panels show the perturbed part. 
(a)

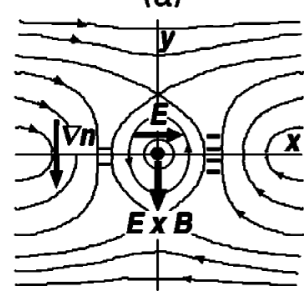

(b)

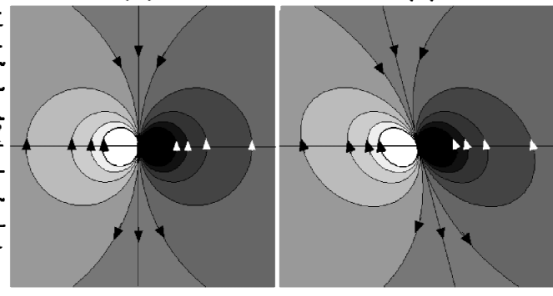

FIG. 6. (a) Streamlines of background electrons in the moving frame centered on a point vortex that is driven downward along the gradient of background density. (b) The perturbed vorticity distribution (3) for $A=0$. (c) The perturbed vorticity distribution (2) for $A \neq 0$.

creates the perturbed density distribution as observed in Fig. 5.

On the basis of the above discussion, we learn the physical meaning of Eq. 4 in Ref. [13], that may be rewritten as

$$
\delta \zeta_{b}=\frac{\Gamma_{v}}{4 \pi} \frac{d \zeta_{b 0} / d r}{A \rho \sin \theta} \ln [1+A t \sin \theta(A t \sin \theta-2 \cos \theta)],
$$

where $\rho=\left(x^{2}+y^{2}\right)^{1 / 2}$, and $\theta=\arctan (y / x)$. The physical picture given in Fig. 6(a) corresponds to the limit of $A=0$, where

$$
\delta \zeta_{b}=-\frac{\Gamma_{v} d \zeta_{b 0} / d r}{2 \pi} \frac{t \cos \theta}{\rho} .
$$

Equation (3) represents a dipole configuration of vorticity as shown in Fig. 6(b) that produces velocity field orienting to negative $y$ in the same way as in Fig. 6(a). Note that the induced space charge density in Fig. 6(a) is equivalent to the vorticity distribution in Fig. 6(b). It is also noteworthy that $\delta \zeta_{b}$ increases linearly in time. It is related to the observations given in Fig. 3 that in the initial phase (small $|A t|)$ the radial velocity of the vortex increases in time. If $A \neq 0$, Eq. (2) tells that the axis of the dipole structure tilts to the direction $\theta=\arctan (2 / A t)$, as drawn in Fig. 6(c). The tilting limits a further increase of the radial velocity and reduces the azimuthal velocity in the laboratory frame.

The linear theory does not tell how $\delta \zeta_{b}$ evolves to form a spiral structure in $\zeta_{b}$ as the vortex changes its location in the laboratory frame. A quantitative analysis of the images as shown in Fig. 5 indicates that $\left|\delta n_{b} / n_{b}\right|=\left|\delta \zeta_{b} / \zeta_{b}\right|$ amounts to several tens of percent in the wake of the vortex as $\Gamma_{v} \propto N_{v}$ increases. The long-lasting wake itself is associated with the conservation of vorticity in the Euler fluid, but the process determining the modulation level remains as an important problem to be explored theoretically. Observations as in Fig. 5 also indicate that the spiral structure rotates differentially with the vortex. The rotation of the modulated background generates an oscillating azimuthal electric field at the position of the vortex and drives it radially in and out. The oscillatory trajectories of a vortex shown in Fig. 3 are a manifestation of this process. Our experimental study indicates the importance of extending the model to the nonlinear stage and to consideration of nonlocal effects in time and space.

In summary, we have experimentally studied dynamics of a point vortex in interaction with a background vorticity of various profiles, and compared the observations with prediction of a theoretical model [13]. The experiment has confirmed essential aspects of the model, but it also indicates several aspects to be studied further beyond the linear analysis as employed for constructing the theoretical model.

One of the authors (Y.K.) thanks D. A. Schecter, Professor Fred Driscoll, and Professor Tom O'Neil for enlightening discussion. This work was supported by the Grant-in-Aid program of the Ministry of Education, Science, Sports and Culture of Japan.

*Email address: m52279@sakura.kudpc.kyoto-u.ac.jp

[1] R. H. Levy, Phys. Fluids 11, 920 (1968).

[2] R. J. Briggs, J. D. Daugherty, and R. H. Levy, Phys. Fluids 13, 421 (1970).

[3] C. F. Driscoll, Phys. Rev. Lett. 64, 645 (1990).

[4] J.H. Malmberg and J.S. deGrassie, Phys. Rev. Lett. 35, 577 (1975).

[5] K. S. Fine et al., Phys. Rev. Lett. 67, 588 (1991).

[6] T. B. Mitchell, C.F. Driscoll, and K. S. Fine, Phys. Rev. Lett. 71, 1371 (1993).

[7] K. S. Fine et al., Phys. Rev. Lett. 75, 3277 (1995).

[8] D. A. Schecter et al., Phys. Fluids 11, 905 (1999).

[9] Y. Kiwamoto et al., J. Phys. Soc. Jpn. 68, 3766 (1999).

[10] G. F. Carnavele et al., J. Fluid. Mech. 233, 119 (1991).

[11] G. M. Reznik, J. Fluid. Mech. 240, 405 (1992).

[12] G. F. Carnavele et al., Phys. Fluids A 3, 1411 (1991).

[13] D. A. Schecter and D. H.E. Dubin, Phys. Rev. Lett. 83, 2191 (1999).

[14] Photocathodes are used by D. Durkin and J. Fajans, Rev. Sci. Instrum. 70, 4539 (1999). 